Session 1647

\title{
Lectures for Internet Courses
}

\author{
Hal Broberg, Paul Lin \\ ECET Department, Indiana University-Purdue University Fort Wayne
}

\begin{abstract}
Many students want an audio/video lecture component with an Internet course and PowerPoint is a good starting point for creating these presentations. Streaming media, using free players, such as RealOne ${ }^{\mathrm{TM}}$ (formerly RealPlayer) provide a method of transmitting audio/video lectures either live, or recorded. Another method is use of Macromedia Director to create individual lecture files which can be downloaded from a website. Director can also be used to create self-loading CD's to provide students with an entire semester of lectures. This paper explains the use of these methods and lessons learned from the courses.
\end{abstract}

\section{Introduction}

A lecture-like presentation may be needed to convey information to students in undergraduate Internet courses. Building Internet courses ${ }^{1,2}$ is a difficult and timeconsuming task ${ }^{3}$. This paper discusses the utility and effectiveness of some of the methods available for dissemination of audiovisual material via the Internet. A source of material to assist the higher education community in use of the Internet is the Joint Information Systems Committee ${ }^{4}$ and related sites. The methods to be discussed here are: creating PowerPoint ${ }^{5}$ audio presentations using WAV files, creating PowerPoint slides

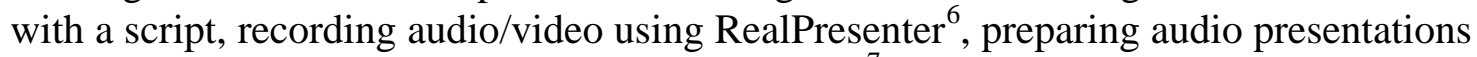
using Powerpoint, then using Macromedia Director ${ }^{7}$ to create a movie, creating slides and audio presentations directly in Macromedia Director, and creating a self-loading CD using Macromedia Director.

These methods have been used in several courses at Indiana University Purdue University, Fort Wayne ${ }^{8}$, where $\mathrm{WebCT}^{9}$ is used for administration of Internet courses. CS/EET 114, an introductory Windows programming course using Visual Basic and EET 205, an introductory assembly language course using the Microchip ${ }^{10}$ PICmicro® assembly language, were offered via the Internet during Fall 2000, Fall 2001, and Spring 2002. EET 302, an introductory control system course was first offered, via the Internet, during Spring 2002. The usefulness and desirability of these pseudo-lecture methods will be described and compared. Lessons learned will also be provided for instructors desiring to create lectures for use in Internet courses.

\section{Using PowerPoint}

As a standard business presentation tool, PowerPoint is readily used to create audio lecture presentations using the Slide Show menu shown in Fig. 1: 


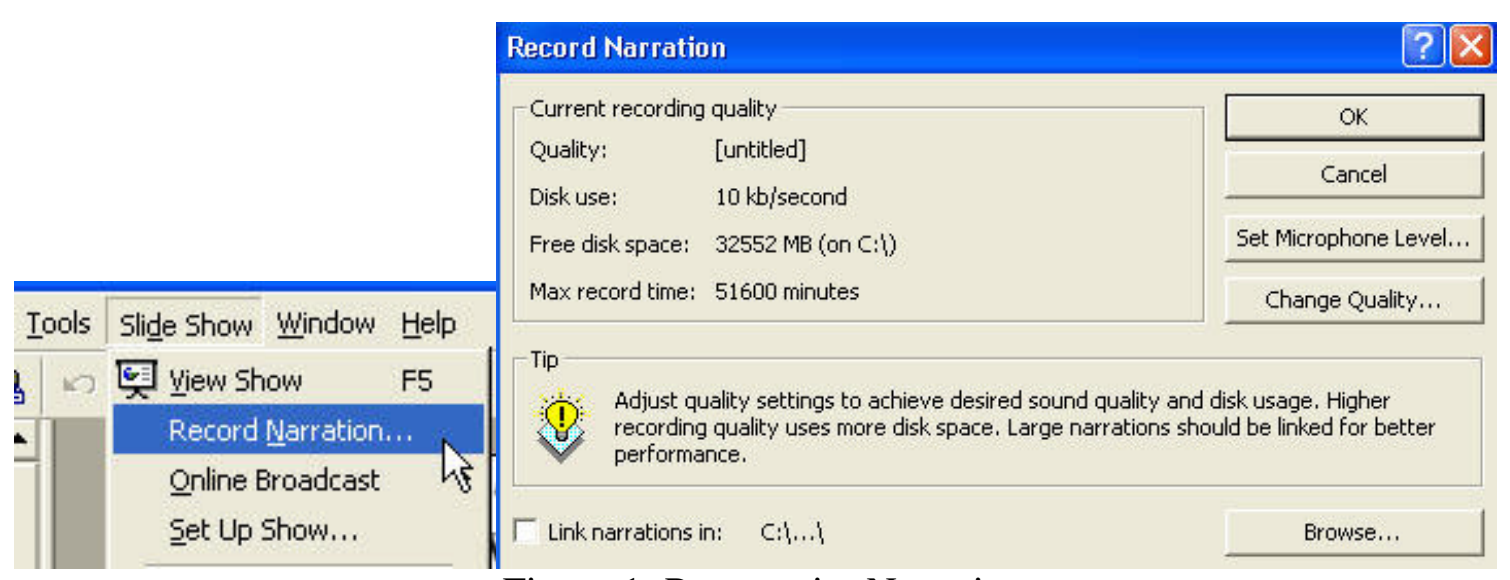

Figure 1: Powerpoint Narration

In the Record Narration window, shown in Fig. 1, the microphone level should be adjusted for normal sound. The 10-kilobit per second rate, shown in Fig. 1, is the default and is satisfactory for voice reproduction. The principal difficulty with PowerPoint audio presentations was found to be the size of the audio (.wav) files created, even with this data rate. The WAV format is an industry standard audio file, but the files are large (about a Megabyte per minute of recording). The resulting lectures are too large for most students to download at home using 56k modems. In addition to this, the WAV files compress very little using WinZip ${ }^{11}$. The large size of these audio/visual files also makes them difficult for students without cable-modem/DSL service to see/listen-to using streaming media.

A solution to this file-size problem using WAV files is to create a screenplay script for each PowerPoint slide and to not record the audio. The script for the audio can be compressed, along with the slides prior to placing the file on a web site for students to download and read. This is satisfactory in many cases and is similar to writing a supplementary book for the course. Many students like to have an audio component similar to classroom lectures that they can watch/listen-to. It was also desirable to use the PowerPoint slides that had already been created for classroom lectures and RealPresenter Plus was designed for this purpose.

\section{Using RealPresenter Plus with PowerPoint}

RealPresenter can be used to create audio/video presentations for streaming via the Internet or an Intranet. One of the RealPresenter windows is shown in Fig. 2.

RealPresenter is approximately $\$ 200$, but provides the capability to stream a presentation to up to 25 users, via an Intranet, from your own computer. RealPresenter also provides the ability to add video, along with audio to your PowerPoint slides. A video of the instructor's head and shoulders was used for the first few lectures during Fall 2000 but the video was dropped due to high bandwidth requirements. A low-resolution, 2frames/second video increased the size of a completed 50 minute lecture file from 50 to 200 Mbytes. This was unacceptable for low-bandwidth $(<=56 \mathrm{k})$ modems, so audio, with PowerPoint slides was used during the remainder of the semester. 


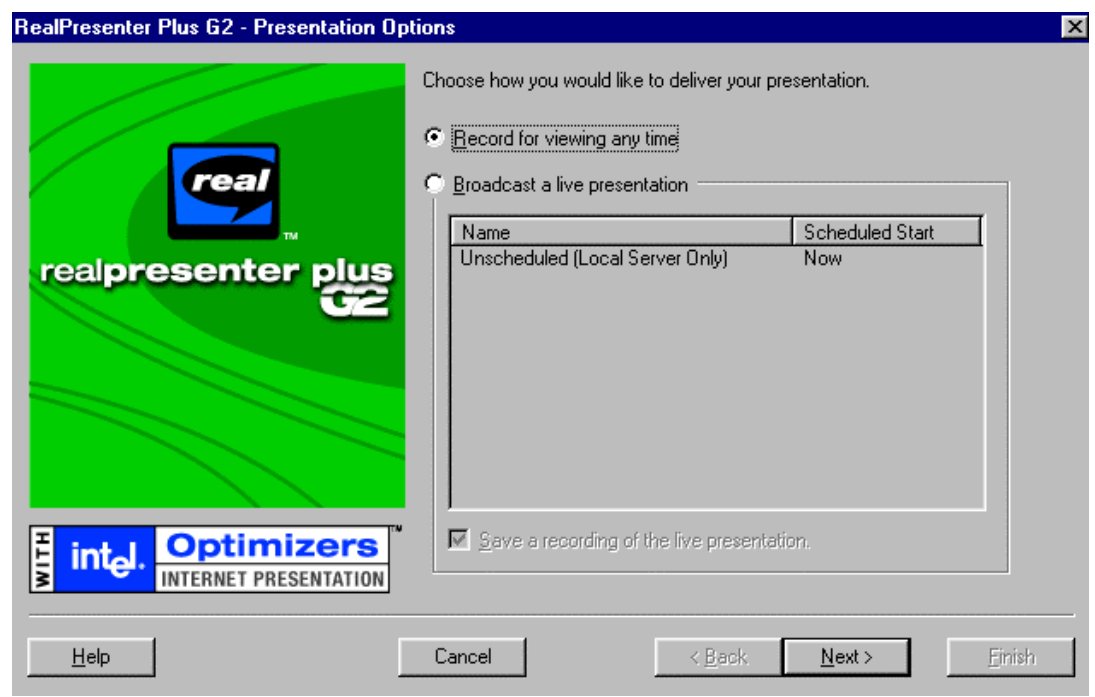

Figure 2: RealPresenter Window

Although even the audio files were large, the classes using RealPresenter Plus to create the files and RealServer ${ }^{12}$ (uploaded via FTP to Indiana University) were quite successful for the great majority of students. A typical streaming download slide in RealPlayer (now called RealOne ${ }^{13}$ ), as seen by a student is shown in Fig. 3. There is commercial advertising associated with the streaming media player shown, however, the lecture

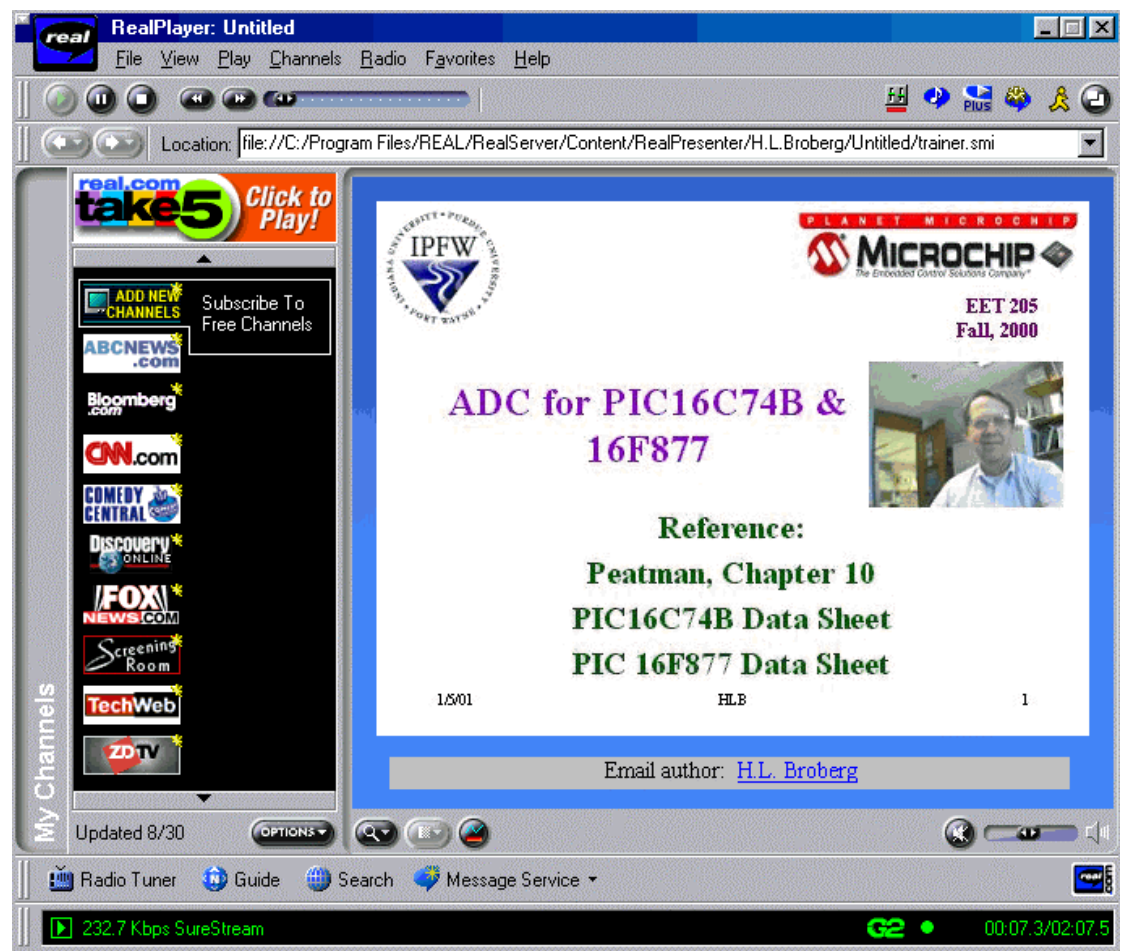

Figure 3: RealPlayer (now RealOne) Window

format was satisfactory for most students. Many 56k modems were satisfactory and the major factor in being able to stream the lectures successfully appeared to be the Internet service provider. A CD of the lecture presentations was made for students with slow 
Internet service providers. Students desiring to print out and read the material could download the PowerPoint slides and script from the class Internet site.

The process of creating RealPresenter lectures was time consuming and the following is a summary of required steps in the creation process:

1. Create PowerPoint ${ }^{\mathrm{TM}}$ slides

2. Write script (optional, but recommended for smooth presentations)

3. Record audio/video presentation

4. Edit presentation \& rerecord as required

5. Place a link to the PPT presentation on the web page for student download.

6. Upload presentation to RealServer (File Transfer Protocol [FTP] was used)

7. Students access the presentation via the class website on WebCT. Students must have RealPlayer (RealOne) loaded on their computer.

\section{Using Macromedia Director with PowerPoint}

Director is a powerful tool in which you can create your own multimedia presentations. It can also import PowerPoint slides and their associated wave files using the Xtras menu, shown in Fig. 4. Note that the PowerPoint slides must be converted to the old PowerPoint 4.0 format prior to importing them and some content may be lost. Also,

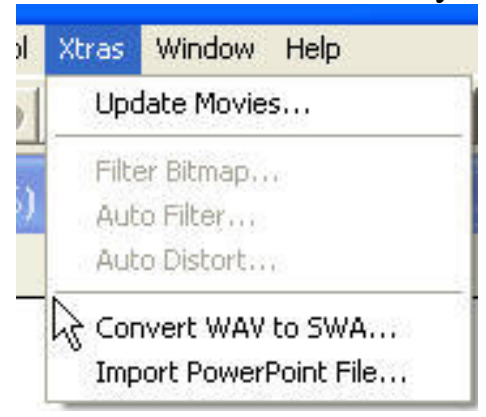

Figure 4: Macromedia Director WAV Conversion Window

WAV files must be imported and converted to SWA (ShockWave Audio) files separately. One advantage of this lengthy conversion process is that the WAV files are compressed by a factor of approximately 10 with no noticeable loss of sound quality. Another advantage is that the resulting Shockwave movie created can be played as if it were an executable file, so the user does not need special software (such as PowerPoint) to play the lecture. A disadvantage is the list price, which is $\$ 1199$, however, a reduced cost educational version is available.

Director can also be used to create a complete semester series of lectures on a selfloading CD. This is labor intensive because the PowerPoint slides and WAV files must be imported and converted to Macromedia format. In addition, learning the Director software and nomenclature is time consuming. Figure 5 shows the main, blank, Macromedia Director window. 


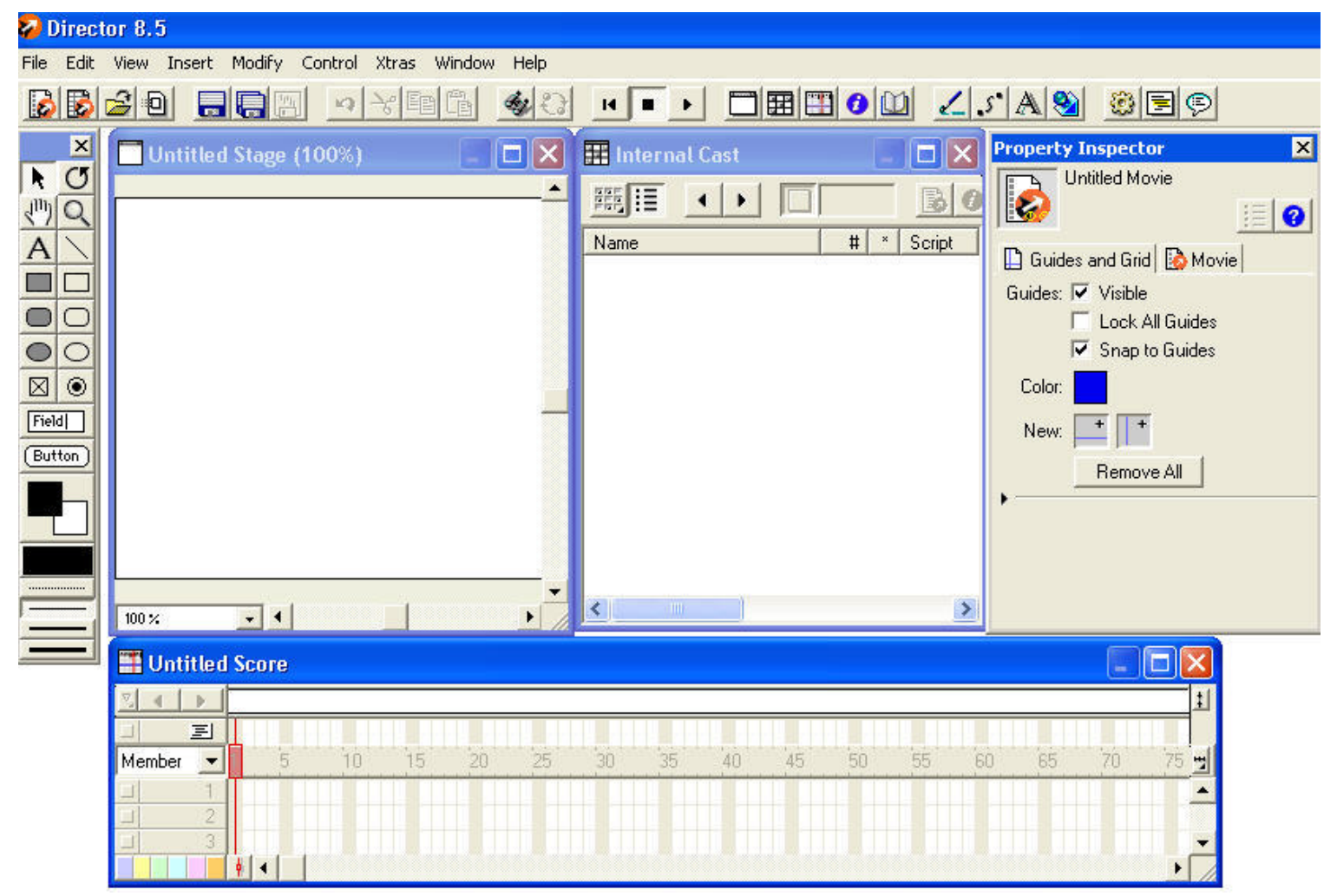

Figure 5: Macromedia Director Main Window

Learning the Director software requires learning terminology such as Stage, Cast, Score, and Script in addition to ensuring that each imported PowerPoint slide is placed correctly as a member of the score. Each SWA (audio) file that was converted from a WAV file must also be individually placed so that it plays when the slide appears on the screen. The following general steps are required to produce a self-loading CD that plays Shockwave movies.

1. Create Powerpoint slides and script

2. Convert to PPT 4.0 (a version from about 1995) and import to Director (Slides can also be created in Director)

3. Record audio in Powerpoint (large. WAV files)

4. Convert to smaller .SWA files

5. Import Audio files and connect them to the imported PPT files

6. Make a cover screen that displays when the CD loads with pointers to each lecture

7. Create Self-Loading CD

The screen that is automatically loaded when the CS/EET $114 \mathrm{CD}$ is placed in the CD reader is shown in Fig. 6. Each of the yellow buttons opens the lecture with the title shown. The lecture numbers are used as part of the assignment on the class syllabus. A similar CD was developed for the EET 205 course. When one of the yellow buttons is selected, the lecture begins. A sample screen from one of the lectures in the EET 205 course is shown in Fig. 7. Note that the screen shown in Fig. 7 looks like a PowerPoint slide and is clean and uncluttered. Rather than a video of the instructor, I placed different still photos on the first page of each lecture to try to retain some connection with the 
student. Also notice in Fig. 7, that there is a button to go back to the Main Menu, which is the menu shown in Fig. 6, and a button to move to the Next slide in the lecture. On subsequent slides there is also a button at the bottom of the screen so the student can move back and review the previous slide. Thus, the student has complete control of what he/she will

\section{CS/EET 114}

Principal Subjects are shown along with (\#Slides/Minutes of Audio) Click on Session to view

\begin{tabular}{|c|c|}
\hline 1. Introduction (13/15) & 14. Review for Quiz \#2 (31/27) \\
\hline 2. A First Program $(17 / 21)$ & 15. Lists and Loops (23/27) \\
\hline 3. More Controls (22/35) & 16. Saving Sequential Files (16/17) \\
\hline 4. Coding for Controls $(12 / 16)$ & 17. Data Arrays (14/15) \\
\hline 5. Chapter 2: Examples (22/19) & 18. User Defined Data Type $(18 / 18)$ \\
\hline 6. Basic Data (12/21) & 19. Drag and Drop (15/14) \\
\hline 7. Calculation and Counting (15/24) & 20. Intro to Graphics (15/14) \\
\hline 8. Review for Quiz \#1 (37/22) & 21. Methods \& Animation (20/13) \\
\hline 9. Decision Making (14/17) & 22. Review for Quiz \#3 (27/26) \\
\hline 10. Operators \& MsgBoxes $(18 / 19)$ & 23. Intro to Object Oriented \\
\hline 11. Menus/Subs/Functions (22/21) & Programming (20/17) \\
\hline 12. Multiple Forms (14/14) & 24. Classes and Collections (21/23) \\
\hline 13. About \& Splash Forms (18/19) & 25. Saving Random Files (17/13) \\
\hline \multicolumn{2}{|c|}{ Press Back or Next to move around in the presentation. Esc to End } \\
\hline
\end{tabular}

Figure 6: Automatically loaded screen for the CS/EET 114 CD

hear and see and can review portions that are difficult. The student must interact with the program to advance the slides. Generally, each of the slides was kept to about a minute of audio, so the student would not become bored. Notice also, that the 25 lectures shown above are not long lectures. All material from the classroom lecture courses was covered in far less time when reading from a script because writing on the board, moving overheads to the screen, and other normal, time-consuming, classroom activities were unnecessary. Additional material, such as lab manuals, assignments, and programs was also included on the $\mathrm{CD}$. The high compression of the Director program reduced an entire semester of lectures for each course to approximately 65-70 Mbytes of space, so each CD had room for much additional material for the class.

\section{Lessons Learned}

Some general items to consider prior to selecting a method of pseudo-lecture presentation via the Internet are shown below.

1. If you intend to use one of these methods, try to obtain a grant or release time to do the planning and preparation required to convert a course to the Internet. 
During this time you can choose and learn the software you will use and prepare the initial slides required for the course.

2. If you want to use RealPresenter or a similar streaming media system, be aware of

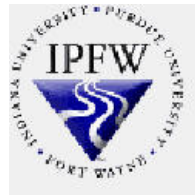

Chapter 4

Interrupts

Timer Interrupts

Interrupt Service Routines

Timer0, Timer1, Timer2

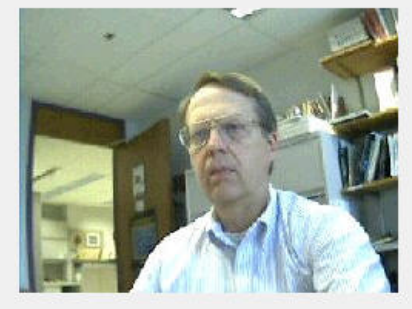

HLB

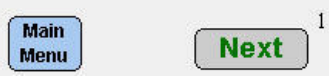

Figure 7: Introductory Screen for an EET205 Presentation

the bandwidth requirements of students who do not have a fast Internet connection. Some students with standard telephone modems ( $<=56 \mathrm{k})$ will have difficulty receiving the streaming media without interruptions and delays. Also, for a Streaming media presentation, ensure that you have backup methods for students who cannot receive the presentation directly. For instance, CD's can be burned for students to check out and compressed PowerPoint files can be made available on the class Internet site for students to download.

3. The use of CD's appears to be successful and students were satisfied with the presentations. A CD that contains a semester of lectures enables the instructor to spend the requisite time on the many other aspects of an Internet course. Some of the time-consuming tasks include email (expect to send an average of 30-40 emails per student per course), bulletin boards, chat rooms and Internet testing. A tabulation of numerical student evaluations, collected via an anonymous web survey, for the courses showed results that were similar to the instructor's evaluations for an in-class lecture. Students also submitted written comments via the anonymous Web survey. Selected written comments received for the question "What did you like most about this course?" are listed below:

convenience

ability to complete projects according to my own pace and schedule from the privacy and comfort of my home

having the opportunity to take this class via the internet

that it was offered via the internet

liked the slide lectures

most questions (from lectures) were answered quickly 
being able to access all information for this class via the internet

the CD lectures were very helpful

I thought the lecture $\mathrm{CD}$ was helpful

having lectures on $\mathrm{CD}$ to review as often as needed.

\section{Summary}

Each of the methods discussed is more time consuming than a "normal" classroom lecture, but once created, the streaming lectures or CD can be used for several semesters, which amortizes the effort. Bandwidth considerations are paramount when streaming media is used because most students use 56k modems at home. The use of compressed PowerPoint slides and scripts available for student download proved effective, but for an audio, lecture-like, component, creation of a lecture CD appears to be the best method currently available. Internet courses using these lecture methods have been well received by students as shown by student comments from an anonymous survey.

\footnotetext{
${ }^{1}$ Building Learning Communities in Cyberspace, Effective Strategies for the Online Classroom, R. M. Palloff and K. Pratt, Jossey-Bass Publishers, 1999

${ }^{2}$ Creating the Virtual Classroom, Distance Learning with the Internet, L. Porter, Wiley, 1997

3 Issues in Web-Based Pedagogy, A Critical Primer, Robert A. Cole (editor), Chapter 15 "The "Time Factor in On-line Teaching: Implications for Faculty and Their Universities” by Claudine SchWeber, Greenwood Press, 2001

${ }^{4}$ JISC Technology Applications Programme (JTAP), http://www.jtap.ac.uk/

${ }^{5}$ http://www.microsoft.com/office/powerpoint/default.htm

${ }^{6} \mathrm{http} / / / \mathrm{www}$. realnetworks.com/products/presenter/index.html

7 http://www.macromedia.com/software/director/

8 http://www.ipfw.edu/

9 http://www.webct.com/

${ }^{10} \mathrm{http}: / /$ www.microchip.com

11 http://www.winzip.com/

12 http://www.realnetworks.com/products/media_delivery.html

13 http://www.real.com/realone/index.html
}

\section{HAL BROBERG}

Hal received his PE license in Indiana in 1988 and his $\mathrm{PhD}$ in Engineering (EE) in 1993. His research area is servo systems and he consults for ITT (Aerospace-Communications) on weather satellite servos and has a Patent Pending. He is an Associate Professor of EET, Interim Associate Dean of the school of Engineering, Technology, and Computer Science and a senior member of and program evaluator for IEEE.

\section{PAUL I-HAI LIN}

Paul is Associate Professor and Chair of the Electrical and Computer Engineering Technology, Indiana Purdue University Fort Wayne. He has been with Purdue University since 1985 and is a registered Professional Engineer (EE) in California and Indiana. Lin's current interests include Web engineering, distributed intelligent control of embedded real-time systems, and sensors in industrial control applications. 